\title{
Recovery from ICU-acquired weakness; do not forget the respiratory muscles!
}

\author{
Rik Gosselink, Daniel Langer
}

Skeletal muscle dysfunction acquired during critical illness (intensive care unit (ICU)-acquired weakness, ICUAW) plays a pivotal role in clinical outcomes such as liberation from mechanical ventilation, ICU length of stay, hospital length of stay, physical function and mortality. ${ }^{1}{ }_{2}^{2}$ ICUAW is a common complication of critical illness with a complex aetiology, ${ }^{3}$ affecting both limb muscles as well as respiratory muscles. The decline in muscle mass is approximately $2 \%-4 \%$ per day in the first week of ICU stay. ${ }^{45}$ Loss of limb muscle mass is more pronounced in patients with multiple organ failure, ${ }^{4}$ while a rapid decline in diaphragm muscle strength and thickness is associated with sepsis $^{6}$ and low diaphragm contractile activity. ${ }^{5}$ Strategies to prevent or treat ICUAW are scarce and mostly focused on the treatment or reduction of risk factors associated with ICUAW (sepsis, hyperglycaemia, catabolism, neuromuscular blockers and corticosteroids). ${ }^{3}$ In addition, immobility and inactivity contribute considerably to muscle atrophy: 'mechanical silencing' has been identified as an important contributor to the loss of contractile properties. ${ }^{7}$ Therefore, reversing inactivity of the muscle should have the potential to prevent, reverse or ameliorate muscle wasting.

The focus of rehabilitation in patients who are critically ill is on the prevention and treatment of ICUAW, oftentimes specifically targeting lower limb muscle function. Early mobilisation, transferring patients from the bed to the chair, weightbearing, walking, bed cycling and neuromuscular electrical stimulation are the most common modalities successfully applied to ameliorate limb muscle weakness and functional status. ${ }^{8}$ It is unclear as to why the respiratory muscles are only very rarely addressed in these programmes. Respiratory muscle dysfunction

Faculty of Kinesiology and Rehabilitation Sciences, Division of Respiratory Rehabilitation, Department Rehabilitation Sciences KU Leuven, University Hospitals Leuven, Leuven, Belgium

Correspondence to Professor Rik Gosselink, Faculty of Kinesiology and Rehabilitation Sciences, Department Rehabilitation Sciences KU Leuven, Division of Respiratory Rehabilitation, University Hospitals Leuven, Tervuursevest 101, Leuven 3000, Belgium;

Rik.Gosselink@faber.kuleuven.be in patients who are mechanically ventilated is observed in $80 \%$ of patients with ICUAW. ${ }^{9}$ Goligher et al documented that a lower contractile activity of the diaphragm during mechanical ventilation was associated with further reduction of diaphragm thickness. ${ }^{5}$ This observation supports the idea that well-balanced intermittent loading of the respiratory muscles during the process of mechanical ventilation might be beneficial to prevent or ameliorate muscle atrophy. Indeed, modalities inducing (intermittent) loading of the respiratory muscles such as spontaneous breathing trials and early mobilisation have been shown to increase muscle strength $^{10}$ and to shorten the duration of mechanical ventilation, ${ }^{11}$ respectively.

However, $15 \%-20 \%$ of patients fail successful liberation from mechanical ventilation. ${ }^{12}$ Inadequate ventilatory drive, increased work of breathing and weakness of the respiratory muscles are likely to contribute to weaning failure. ${ }^{13}$ The inability to breathe spontaneously relates to an imbalance between load on the respiratory muscles and the capacity of the respiratory muscles. ${ }^{14}$ Indeed, high rates of respiratory muscle effort (ratio of workload and muscle capacity $\left(\mathrm{P}_{\mathrm{I}} / \mathrm{P}_{\text {Imax }}\right)$ ) are a major cause of ventilator dependency and predict the outcome of successful liberation from mechanical ventilation. ${ }^{15}$ In patients at risk for ventilatory failure following extubation, unloading of the respiratory muscles with non-invasive ventilation has been shown successful in facilitating discontinuation of invasive mechanical ventilation. ${ }^{16}$ Surprisingly, little attention has been given to specific interventions to enhance strength and endurance of the respiratory muscles. Indeed, daily intermittent inspiratory loading with six to eight contractions repeated in three to four series at moderate to high intensity was safe, improved inspiratory muscle strength and weaning success in patients with difficult weaning. ${ }^{17}$ One of the challenges of these studies is that patients who might benefit from the intervention are oftentimes not sufficiently able to participate in the training sessions.

Given the difficulties of inspiratory muscle training (IMT) during mechanical ventilation as well as the fact that weakness persists after successful extubation, ${ }^{18}$ targeted training approaches following successful extubation might be warranted. This question was adequately addressed by Bissett and colleagues. ${ }^{19}$ The authors provided IMT for 2 weeks in patients who were successfully weaned from mechanical ventilation. This approach might boost the rehabilitation allowing better coping with the ventilatory demands during walking, cycling, activities of daily living, improve quality of life and reduce dyspnoea. As expected, $\mathrm{P}_{\text {Imax }}$ improved significantly in the intervention group ( $17 \%$ predicted vs $6 \%$ pred in the control group), but no improvements in inspiratory muscle endurance, physical function and dyspnoea were observed. Though disappointing at first glance, several reasons could have been involved in the lack of transfer effects to exercise performance and dyspnoea. First, 2 weeks of training may be too short to improve exercise performance and dyspnoea. To obtain these effects, a rehabilitation programme combining limb muscle training and respiratory muscle training is probably warranted. Since no data were collected on the progress of training intensity during the 2 weeks, it is unknown how patients tolerated the increased training intensity. Improvements in $\mathrm{P}_{\mathrm{Imax}}$ varied substantially and increased specifically in patients with relatively preserved $\mathrm{P}_{\text {Imax }}$, while patients with very low $\mathrm{P}_{\text {Imax }}$ showed modest improvements. This might be related to the fact that a low training intensity could not be adequately set. The minimal inspiratory resistance at the Threshold device is $9 \mathrm{~cm} \mathrm{H}_{2} \mathrm{O}$, and this might have been too high for very weak patients. In general, threshold loading might be less optimal compared with (electronic) tapered flow-resistive loading. ${ }^{20}$ The latter type of inspiratory muscle loading starts at very low intensity $\left(4 \mathrm{~cm} \mathrm{H}_{2} \mathrm{O}\right)$. In addition, the attenuating resistance over the inspiratory cycle allows training with a larger tidal volume, higher power per breath and less dyspnoea. ${ }^{20}$ In patients with COPD, this device has been shown to be more effective in improving inspiratory muscle strength and endurance. ${ }^{20}$ The lack of improvement in muscle endurance in the study of Bissett et al might also be related to the incremental loading protocol that was applied. ${ }^{19}$ In general, a constant load protocol is a more sensitive measure and might reflect better endurance capacity than the incremental loading protocol. Moreover, in the present study, patients were not specifically selected with inspiratory muscle weakness, the considered target population for such interventions. On average, patients had reduced $\mathrm{P}_{\text {Imax }}$, but 
several patients had at the time of enrolment (near) normal respiratory muscle strength. These patients are probably not appropriate candidates for respiratory muscle training interventions. Furthermore, it is questionable whether the training device used by the authors was capable of providing a sufficiently high training stimulus for these patients with near-normal respiratory muscle strength.

Finally, the mortality in the experimental group (12\%) was anticipated in the design of the study $(12.5 \%)$, but the control group had a (borderline statistically significant) lower mortality. This unexpected difference in mortality was neither related to the IMT sessions nor to respiratory complications. The authors suggested that the heterogeneity and the size of the study group might explain this finding. The higher mortality in the IMT group is in contrast with the findings reported in a recent meta-analysis on the effects of IMT to facilitate liberation from mechanical ventilation. The authors reported a slightly higher, but not statistically significant, likelihood of survival in the IMT group when pooling data from four studies $(n=242$; relative risk $(95 \%$ CI) of survival: 1.04 (0.96 to 1.13$)) .{ }^{17}$ Weighted mortality rates were $6.4 \%$ in the intervention group and $10.3 \%$ in the control group.

Considering the promising results of the study of Bissett and colleagues and the emerging role of ICUAW, including respiratory muscle weakness, future studies are warranted. Since unsupervised home-based post-ICU rehabilitation has been unsuccessful, ${ }^{21}$ a supervised rehabilitation programme including IMT should be investigated in patients with inspiratory muscle weakness. The outcomes of these studies should specifically focus, in addition to respiratory and limb muscle functions, on dyspnoea, respiratory infections, hospital and ICU stay, hospital and ICU readmission, exercise capacity, functional status and quality of life.

\section{Competing interests None declared.}

Provenance and peer review Commissioned; externally peer reviewed.

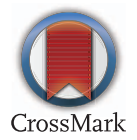

To cite Gosselink R, Langer D. Thorax 2016;71:779780.

Published Online First 21 July 2016

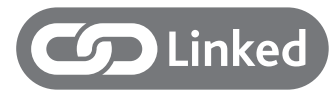

http://dx.doi.org/10.1136/thoraxjnl-2016-208279

Thorax 2016;71:779-780.

doi:10.1136/thoraxjnl-2016-208835

\section{REFERENCES}

1 Hermans G, Van Mechelen H, Clerckx B, et al. Acute outcomes and 1-year mortality of intensive care unit-acquired weakness. A cohort study and propensity-matched analysis. Am J Respir Crit Care Med 2014;190:410-20.

2 Wieske L, Dettling-Ihnenfeldt DS, Verhamme C, et al. Impact of ICU-acquired weakness on post-ICU physical functioning: a follow-up study. Crit Care 2015;19:196.

3 Friedrich $\mathrm{O}$, Reid MB, Van den Berghe $\mathrm{G}$, et al. The sick and the weak: neuropathies/myopathies in the critically ill. Physiol Rev 2015:95:1025-109.

4 Puthucheary ZA, Rawal J, McPhail M, et al. Acute skeletal muscle wasting in critical illness. JAMA 2013;310:1591-600

5 Goligher EC, Fan E, Herridge MS, et al. Evolution of diaphragm thickness during mechanical ventilation. Impact of inspiratory effort. Am J Respir Crit Care Med 2015;192:1080-8.

6 De Jonghe B, Bastuji-Garin S, Durand M-C, et al. Respiratory weakness is associated with limb weakness and delayed weaning in critical illness. Crit Care Med 2007:35:2007-15.

7 Llano-Diez M, Renaud G, Andersson M, et al. Mechanisms underlying ICU muscle wasting and effects of passive mechanical loading. Crit Care 2012;16:R209.

8 Castro-Avila AC, Serón P, Fan E, et al. Effect of early rehabilitation during intensive care unit stay on functional status: systematic review and meta-analysis. PLOS ONE 2015;10:e0130722.

9 Jung B, Moury PH, Mahul M, et al. Diaphragmatic dysfunction in patients with ICU-acquired weakness and its impact on extubation failure. Intensive Care Med 2016;42:853-61.

10 Gayan-Ramirez G, Testelmans D, Maes K, et al. Intermittent spontaneous breathing protects the rat diaphragm from mechanical ventilation effects. Crit Care Med 2005:33:2804-9.

11 Schweickert WD, Pohlman MC, Pohlman AS, et al. Early physical and occupational therapy in mechanically ventilated, critically ill patients: a randomised controlled trial. Lancet 2009;373:1874-82.

12 McConville JF, Kress JP. Weaning patients from the ventilator. N Engl J Med 2012;367:2233-9.

13 Peñuelas 0 , Frutos-Vivar $F$, Fernández $C$, et al. Characteristics and outcomes of ventilated patients according to time to liberation from mechanical ventilation. Am J Respir Crit Care Med 2011;184:430-7.

14 Goldstone J, Moxham J. Assisted ventilation. 4. Weaning from mechanical ventilation. Thorax 1991:46:56-62.

15 Vassilakopoulos T, Zakynthinos S, Roussos C. The tension-time index and the frequency/ tidal volume ratio are the major pathophysiologic determinants of weaning failure and success. Am J Respir Crit Care Med 1998;158: 378-85.

16 Nava S, Gregoretti C, Fanfulla F, et al. Noninvasive ventilation to prevent respiratory failure after extubation in high-risk patients. Crit Care Med 2005:33:2465-70.

17 Elkins M, Dentice R. Inspiratory muscle training facilitates weaning from mechanical ventilation among patients in the intensive care unit: a systematic review. J Physiother 2015;61:125-34.

18 Chang AT, Boots RJ, Brown MG, et al. Reduced inspiratory muscle endurance following successfu weaning from prolonged mechanical ventilation. Chest 2005;128:553-9.

19 Bissett BM, Leditschke IA, Neeman T, et al. Inspiratory muscle training to enhance recovery from mechanical ventilation: a randomised trial. Thorax 2016;71:812-9.

20 Langer $\mathrm{D}$, Charususin N, Jácome C, et al. Efficacy of a novel method for inspiratory muscle training in people with chronic obstructive pulmonary disease. Phys Ther 2015;95:1264-73.

21 Elliott D, McKinley S, Alison J, et al. Health-related quality of life and physical recovery after a critical illness: a multi-centre randomised controlled trial of a home-based physical rehabilitation program. Crit Care 2011;15:R142. 